\title{
Adverse Event Related to Commutability
}

National Cancer Institute

\section{Source}

National Cancer Institute. Adverse Event Related to Commutability. NCI Thesaurus. Code C139525.

The adverse event occurred because the material being used to calibrate or assess IVD performance did not have similar properties to those of human samples, leading to inappropriate bias and erroneous results. Examples of materials include: reference materials, calibrators, proficiency testing samples. 